\title{
On Generalized Langevin Dynamics and the Modelling of Global Mean Temperature.
}

\author{
Nicholas W. Watkins ${ }^{1,2,3}$, Sandra C. Chapman ${ }^{1}$, Aleksei Chechkin ${ }^{4,5}$, Ian \\ Ford $^{6}$, Rainer Klages ${ }^{7}$, and David A. Stainforth ${ }^{2,1}$ \\ 1 University of Warwick, Coventry CV4 7AL, UK \\ nickwatkins62@fastmail.com, \\ WWW home page: \\ http://warwick.ac.uk/fac/sci/physics/research/cfsa/people/watkins/ \\ 2 London School of Economics and Political Science, London WC2A 2AE, UK \\ 3 The Open University, Milton Keynes MK7 6AA, UK \\ 4 Akhiezer Institute for Theoretical Physics, Kharkov, Ukraine \\ 5 Institute for Physics and Astronomy, University of Potsdam, Germany \\ ${ }^{6}$ University College London, London WC1E 6BT, UK \\ 7 Queen Mary University of London, London E1 4NS, UK
}

\begin{abstract}
Climate science employs a hierarchy of models, trading the tractability of simplified energy balance models (EBMs) against the detail of Global Circulation Models. Since the pioneering work of Hasselmann, stochastic EBMs have allowed treatment of climate fluctuations and noise. However, it has recently been claimed that observations motivate heavy-tailed temporal response functions in global mean temperature to perturbations. Our complementary approach exploits the correspondence between Hasselmann's EBM and the original mean-reverting stochastic model in physics, Langevin's equation of 1908. We propose mapping a model well known in statistical mechanics, the Mori-Kubo Generalised Langevin Equation (GLE) to generalise the Hasselmann EBM. If present, long range memory then simplifies the GLE to a fractional Langevin equation (FLE). We describe the corresponding EBMs that map to the GLE and FLE, briefly discuss their solutions, and relate them to Lovejoy et al's new Fractional Energy Balance Model (FEBE).
\end{abstract}

Keywords: Climate sensitivity, Hasselmann model, Langevin equation, fractional derivative, long-range dependence.

\section{Introduction and aims of paper}

The importance of modelling the response of the Earth's climate to external perturbations, whether anthropogenic or solar, and internal fluctuations, is well recognised. It has been addressed by models with many complementary levels of complexity [1], from general circulation models to very simplified energy balance models 2]. Key steps included the first deterministic EBMs, and Hasselmann's development 3415 of these into a stochastic differential equation with added white noise. The Hasselmann model was far from the last word in stochastic 
modelling for climate applications, however, and a review of progress in this area was given by the contributors to a recent book [6. Of particular note has been the extensive study of multivariate stochastic models and statistical inference (e.g. 7]), and stochastic modelling 80 in climate science using the MoriZwanzig formalism which provides a very general mathematical framework for the Langevin equation 10:11. Some of this work has benefitted from a formal equivalence between the Hasselmann model and the driven Langevin equation of statistical mechanics, and much of it has been done while still assuming that the noise terms are spectrally white. This greatly eases the mathematical tractability by linking the problem to the most mature parts of stochastic calculus (e.g. [11). However there have long been physically-motivated arguments for making the driving noise term itself spectrally red rather than white. In particular Leith argued more than 25 years ago 12 that: "unfortunately there is evidence that [a model of the white noise-driven Langevin type] would be unsatisfactory to capture some of the low frequency phenomena observed in the atmosphere. This is referred to as the infrared climate problem and appears to be caused by nonlinear interactions of the chaotic internal weather frequencies that potentially induce a "piling-up" of extra variance at the low frequencies".

More recently evidence has been given for long-range dependence (LRD, [13]) in the climate system based on " $1 / \mathrm{f}$ " power spectra and other diagnostics 14. An alternative approach to using a " $1 / \mathrm{f}$ " noise term in a stochastic EBM has been to propose non-exponential, long-tailed response functions in the time domain. These were introduced by Rypdal[15] in a fractional Brownian setting. This pioneering paper was followed by further work from the Tromso group, notably [16] and [17. More recently several papers by Lovejoy and co-workers (e.g. 18]) have demonstrated the utility of a fractional Gaussian model (StocSIPS) for climate prediction on monthly and longer time scales.

As Leith's comments implied, however, the physical arguments for extending the Hasselmann formalism are much more general than those which motivate LRD, and there are many other well-known features of the climate system [6]14] including periodicities and apparent spectral breaks that also need to be captured. So an ideal but still simple stochastic EBM would permit a more general range of dependency structures than either just the shortest possible range (Markovian) or longest range (LRD) behaviours, while allowing both as limiting cases. Just such a formalism has long been available in statistical mechanics [10|19]20|21|22, the Mori-Kubo generalised Langevin equation. This is a natural extension of the Langevin equation whereby an integral over a kernel replaces its constant damping term.

In this paper we propose extending the Hasselmann model by this GLEinspired route, and show what the analogous generalised Hasselmann equation would be. We then recap how taking a power-law form for the damping kernel arrives at the fractional Langevin equation studied by researchers in topics including anomalous diffusion, and write down a corresponding fractional Hasselmann equation. Fractionally integrating both sides of this allows us to identify 
how Lovejoy et al's new 23124 fractional energy balance equation (FEBE) relates to our scheme.

\section{Deterministic and stochastic energy balance models.}

The simplest energy balance models are deterministic. They describe the (assumed linear) change $\Delta T(t)$ in the Earth's global mean temperature $T(t)$ away from its radiative equilibrium value $T_{0}$ (i.e. the anomaly in GMT) due to changes in the net radiative forcing $F(t) . \Delta T$ is multiplied by the effective heat capacity $C$ to give the change $\Delta Q$ in the heat content of the earth system, while the feedbacks in the climate system are lumped into a single parameter $\lambda$, to give a one-dimensional deterministic EBM:

$$
\frac{d \Delta Q}{d t}=C \frac{d \Delta T}{d t}=-\lambda \Delta T(t)+F(t)
$$

Hasselmann's model was a key step in improving the realism of this EBM, by incorporating delta correlated ("white") noise $\xi(t)$ :

$$
<\xi(t) \xi\left(t+t^{\prime}\right)>=\sigma_{Q}^{2} \delta\left(t-t^{\prime}\right)
$$

giving rise to what we will refer to as the driven Hasselmann equation (DHE):

$$
\left(C \frac{d}{d t}+\lambda\right) \Delta T(t)=F(t)+\xi(t)
$$

\section{The Hasselmann-Langevin correspondence}

The DHE is the simplest possible Markovian stochastic linear EBM, and as such, isomorphic to the driven Langevin equation (DLE) of statistical mechanics 26 20, for the velocity of a particle executing Brownian motion:

$$
\left(M \frac{d}{d t}+\eta\right) V(t)=F(t)+\xi(t)
$$

where we followed the convention of [26] that a stochastic variable is upper case while known values of the variable are lower case. The correspondence is well-known in climate science, and can be expressed via a lookup table:

Table 1.

\begin{tabular}{|c|c|c|c|c|c|}
\hline Quantity & Symbol & Units & Quantity & Symbol & Units \\
\hline Velocity & $V(t)$ & {$\left[\mathrm{ms}^{-1}\right]$} & GMT anomaly & $\Delta T(t)$ & {$[K]$} \\
Initial $V$ & $v_{0}$ & {$\left[\mathrm{~ms}^{-1}\right]$} & Initial $\Delta T$ & $\Delta T_{0}$ & {$[K]$} \\
Drift $V$ & $v_{d}$ & {$\left[\mathrm{~ms}^{-1}\right]$} & & $F / \lambda$ & \\
Mass & $M$ & {$[\mathrm{~kg}]$} & Heat capacity & $C$ & {$\left[J K^{-1} \mathrm{~m}^{-2}\right]$} \\
Damping rate & $\eta / M$ & {$\left[\mathrm{~s}^{-1}\right]$} & & $\lambda / C$ & {$\left[\mathrm{~s}^{-1}\right]$} \\
Noise strength & $\sigma_{v} / M$ & {$\left[\mathrm{~ms}^{-3 / 2}\right]$} & & $\sigma_{Q} / C$ & {$\left[K s^{-1 / 2}\right]$} \\
\hline
\end{tabular}


It should be noted that the Hasselmann model is sometimes used without a deterministic forcing term $F(t)$ (e.g. [27]). We will refer to this as the undriven Hasselmann equation (UHE):

$$
\left(C \frac{d}{d t}+\lambda\right) \Delta T(t)=\xi(t)
$$

and note that it is of course in correspondence with the undriven Langevin equation

$$
\left(M \frac{d}{d t}+\eta\right) V(t)=\xi(t)
$$

whose solution[11/26]28] is the well-known Ornstein-Uhlenbeck process .

In standard texts such as 28 it is shown that in the undriven $F=0$ case, and for a solution started at $t=0$ with velocity $v_{0}$, the velocity process of the ULE is

$$
V(t)=v_{0} e^{-(\eta / M) t}+\frac{1}{M} \int_{0}^{t} e^{-(\eta / M)(t-\tau)} \xi(\tau) d \tau
$$

The equivalent solution for the temperature process in the UHE is well-known to be:

$$
\Delta T(t)=\Delta T_{0} e^{-(\lambda / C) t}+\frac{1}{C} \int_{0}^{t} e^{-(\lambda / C)(t-\tau)} \xi(\tau) d \tau
$$

As noted by [28 the use of the alternative initial condition whereby the evolution starts at $t=-\infty$ means that the first term in the above can be dropped, giving

$$
V(t)=\frac{1}{M} \int_{-\infty}^{t} e^{-(\eta / M)(t-\tau)} \xi(\tau) d \tau
$$

and

$$
\Delta T(t)=\frac{1}{C} \int_{-\infty}^{t} e^{-(\lambda / C)(t-\tau)} \xi(\tau) d \tau
$$

respectively. The extension of the solution to the case with a nonzero deterministic component of forcing is discussed e.g. by [17.

\section{Non-Markovian models}

We noted in the introduction, however, that in both condensed matter physics and climate science we may not always (e.g. 1029]) be entitled to make the (often very good) approximation that the noise term $\xi(t)$ is white and that the response function is exponential. Debate continues 18 in climate research about when a fluctuation-dissipation theorem applies (or a priori could apply), while in condensed matter applications both internal and external noises are studied 2122], so will not prescribe the power spectrum of $\xi(t)$ in what follows. Since the 1960s one standard way to handle such cases in statistical mechanics 
has been to use the full GLE rather than the Langevin equation which approximates it. In use the initial time $a$ is often be taken to be 0 or $-\infty$ as discussed by e.g. [10]. Following the notation of [30] but with $U^{\prime}(x)=F(t)$ we have

$$
M \frac{d}{d t} V(t)+M \int_{a}^{t} \gamma(t-\tau) V(\tau) d \tau=F(t)+\xi(t)
$$

In the special case that the response is instantaneous and has no memory we have that the damping kernel $\gamma(t)=(\eta / M) \delta(t)$ and the ordinary DLE is retrieved.

The relevance of the GLE has been explicitly noted in climate science 31. but as far as we know it has not been proposed directly as an extension of the Hasselmann EBM. By means of the correspondence used previously we now argue that a generalised Hasselmann equation (GHE) should be:

$$
C \frac{d}{d t} \Delta T(t)+C \int_{a}^{t} \gamma(t-\tau) \Delta T(\tau) d \tau=F(t)+\xi(t)
$$

which would become the familiar Hasselmann equation in the limit $\gamma(t)=$ $(\lambda / C) \delta(t)$.

We are not aware of a more general solution than what is available in the Mori-Zwanzig approach for the above equations for data-derived $F(t)$ of the type encountered in climate modelling. Sophisticated numerics are now available, however (e.g. 32]).

\section{Fractional non-Markovian models}

A special case of the GLE which has received interest (e.g. 2030 3333536]) because of its relationship to anomalous diffusion is when the damping kernel $\gamma(t)$ is chosen to be a power law $\gamma(t) \sim t^{-\alpha}$ (e.g. 3033]). The integral operator term in the GLE can then be replaced (e.g. 3037]) by a Riemann-Liouville fractional derivative with respect to time:

$$
\int_{0}^{t} d \tau \frac{1}{(t-\tau)^{\alpha}} \equiv \gamma_{\alpha} \frac{d^{\alpha-1}}{d t^{\alpha-1}}
$$

where the gamma function in the definition of the derivative was absorbed into $\gamma_{\alpha}$ and again we follow the notational conventions of Lutz 30].

In this case the GLE becomes the fractional Langevin equation (FLE) (e.g. [20/30 33 34 35] ) which in Lutz' notation is:

$$
M \frac{d}{d t} V(t)+M \gamma_{\alpha} \frac{d^{\alpha-1}}{d t^{\alpha-1}} V(t)=F(t)+\xi(t)
$$

while the GHE that we proposed above would become a fractional Hasselmann equation (FHE):

$$
C \frac{d}{d t} \Delta T(t)+C \gamma_{\alpha} \frac{d^{\alpha-1}}{d t^{\alpha-1}} \Delta T(t)=F(t)+\xi(t)
$$


The exponential functions in the solutions of the Langevin equation are known to be a special case of the solution of the FLE in terms of Mittag-Leffler functions (e.g. [20|30|33]), and the analogous expressions would follow for the FHE (see also 24]).

\section{Fractionally integrating the standard fractional non-Markovian models}

The FHE has not so far been studied in the climate literature, but a closely related equation has, Lovejoy et al's 24 2325] FEBE. To see this we can integrate both sides of the FLE and FHE with respect to time, but rather than an integral of unit order we operate with a fractional integral of order $\alpha-1$.

In the FLE case we will refer to the result as the fractionally integrated fractional Langevin equation (FIFLE):

$$
M \frac{d^{2-\alpha}}{d t^{2-\alpha}} V(t)+M \gamma_{\alpha} V(t)=\frac{d^{1-\alpha}}{d t^{1-\alpha}}(F(t)+\xi(t))
$$

The allowed range of $\alpha$ is inherited from that arising from the definition of the Riemann-Liouville fractional integral, so $\alpha>0$. In the special case where the standard 37. FDT for the FLE applies the noise term becomes a fractional Gaussian noise $\xi_{H}$ [37/13 and we can also substitute for $\alpha=2-2 H$ to get

$$
M \frac{d^{2 H}}{d t^{2 H}} V(t)+M \gamma_{2-2 H} V(t)=\frac{d^{2 H-1}}{d t^{2 H-1}}\left(F(t)+\xi_{H}(t)\right)
$$

which will describe the range $1 / 2<H<1$, i.e. when an $\mathrm{BBm}$ which integrates the fractional Gaussian noise term $\xi_{H}$ would be superdiffusive.

The corresponding fractionally integrated fractional Hasselmann equation (FIFHE) would then be

$$
C \frac{d^{2-\alpha}}{d t^{2-\alpha}} \Delta T(t)+C \gamma_{\alpha} \Delta T(t)=\frac{d^{1-\alpha}}{d t^{1-\alpha}}(F(t)+\xi(t))
$$

and if we posit that an FDT having the same functional form as the FLE's applies then we have

$$
C \frac{d^{2 H}}{d t^{2 H}} \Delta T(t)+C \gamma_{2-2 H} \Delta T(t)=\frac{d^{2 H-1}}{d t^{2 H-1}}\left(F(t)+\xi_{H}(t)\right)
$$

which again applies to the range $1 / 2<H<1$. These two versions of the FIFHE are already of a similar form to Lovejoy et al's FEBE [24 25]:

$$
\left(\tau^{H_{L}} \frac{d^{H_{L}}}{d t^{H_{L}}}+1\right) \Delta T(t)=\frac{\tau}{C}(F(t)+\xi(t))
$$


where $H_{L}$ (denoted by " $\mathrm{H}$ " in their papers) is not the usual Hurst exponent $H$ of Mandelbrot's fBm and fGn (e.g. [13/38]), which we used above.

To further pin down the relationship between the FIFHE and FEBE we can identify $H_{L}$ as $1+d$ where $d$ is the memory parameter used in much of the mathematical and statistical literature (e.g. 13]) on stochastic processes which exhibit LRD including $\operatorname{ARFIMA}(p, d, q)$. Thus $H_{L}=1, H=1 / 2, d=0$ is the limit corresponding to Hasselmann's original white noise driven, exponentially relaxing equation, and the range $1 / 2<H_{L}<3 / 2$ is that corresponding to $\mathrm{fBm}$.

If we then identify $1+d$ with our $2-\alpha$, define a time scale $\tau$ via $\tau^{1+d}=\gamma_{\alpha}^{-1}$, and multiply across by $\tau^{1+d} / C$ we see that the FIFHE becomes:

$$
\left(\tau^{1+d} \frac{d^{1+d}}{d t^{1+d}}+1\right) \Delta T(t)=\frac{\tau^{1+d}}{C} \frac{d^{d}}{d t^{d}}(F(t)+\xi(t))
$$

The left hand side of the FIFHE is then seen to be isomorphic to that of the FEBE. The right hand side differs from the FEBE, being a fractional derivative of the sum of the forcing term and a noise, the details of which will depend on those of $\xi$ itself. We stress that this result is independent of whether or not an FDT has yet been assumed.

In the special case where an FDT of the same form as that of the FLE 37. applies we use $\alpha=2-2 H$ to give $d=2 H-1$ and then, as before, define a time scale $\tau$ by $\tau^{2 H}=\gamma_{2-2 H}^{-1}$, and multiply across by $\tau^{2 H} / C$ to give:

$$
\left(\tau^{2 H} \frac{d^{2 H}}{d t^{2 H}}+1\right) \Delta T(t)=\frac{\tau^{2 H}}{C} \frac{d^{2 H-1}}{d t^{2 H-1}}\left(F(t)+\xi_{H}(t)\right)
$$

which, as we remarked in [39] applies in the range $H<1 / 2<1$.

\section{Conclusions}

This paper is a first report on our project [39/40 to use the well-known MoriKubo generalised Langevin equation of statistical mechanics to seek a flexible framework in which the Hasselmann equation and our FIFHE are respectively the short range and long range correlated limits. We intend to further explore the properties of the FHE and FIFHE and their relation to the FEBE in future work, as well as those of the more general GHE for climate.

Acknowledgements We acknowledge valuable interactions with and encouragement from Raphael Calel, Michael Ghil, Valerio Lembo, Shaun Lovejoy, Valerio Lucarini, Nick Moloney, Cecile Penland, Kristoffer Rypdal and Martin Ryp- 
dal. NWW would particularly like to thank the organisers of the Exeter University workshop on Emergent Constraints on 23rd November 2020, questions at which from Peter Cox and Jan Sieber spurred the production of the revised version of this preprint. He also acknowledges support from the Max Planck Society, London Mathematical Laboratory, KLIMAFORSK project number 229754 and ONR grant NICOP - N62909-15-1-N143. NWW and SCC acknowledge the hospitality of Holger Kantz at MPIPKS, Ralf Metzler at the University of Potsdam, Juergen Kurths at PIK and Sid Redner at SFI during the development of this work. SCC acknowledges support from STFC grant ST/T000252/1 and AFOSR grant FA9550-17-1-0054. DAS acknowledges support from the Grantham Research Institute on Climate Change and the Environment, at the London School of Economics, and the ESRC Centre for Climate Change Economics and Policy (CCCEP) (ref. ES/R009708/1).

\section{References}

1. Ghil, M.: A century of nonlinearity in the geosciences. Earth and Space Science. 6, 1007-1042 (2019). doi:10.1029/2019EA000599

2. North, G.R., Cahalan, R.F., Coakley Jr., J. A.: Energy balance climate models. Reviews of Geophysics and Space Physics. 19(1), 91-121 (1981).

3. Hasselmann, K.: Stochastic climate models Part I. Theory. Tellus. 28(6), 473-485 (1976)

4. Frankignoul, C., Hasselmann, K.: Stochastic climate models Part II. Application to sea-surface temperature anomalies and thermocline variability. Tellus. 29(4), 289305 (1977).

5. Lemke, P.: Stochastic climate models Part III. Application to zonally averaged energy models. Tellus. 29(5), 385-392, (1977).

6. Franzke, C., O'Kane, T. (eds.): Nonlinear and Stochastic Climate Dynamics. Cambridge University Press, Cambridge (2017).

7. Penland, C.: Random forcing and forecasting using principal oscillation pattern analysis. Monthly Weather Review. 117, 2165-2185 (1989).

8. Franzke, C. L. E., O'Kane, T. J., Berner, J., Williams, P. D., Lucarini, V.: Stochastic climate theory and modelling. WIREs Clim Change. 6,63-78 (2015). doi:10.1002/wcc.318

9. Gottwald, G., Crommelin, D., Franzke, C.: Stochastic Climate Theory (2017). In 6] pp. 209-240. doi:10.1017/9781316339251.009

10. Zwanzig, R.: Nonequilibrium Statistical Mechanics. Oxford University Press, Oxford (2001).

11. Chorin, A. J., Hald, O. H.: Stochastic Tools in Mathematical Science, Second Edition. Springer Verlag, Berlin (2009).

12. Leith, C.: Climate signal and weather noise, In: Madden, R. A., Katz, R. W. (eds.) Applications of Statistics to Modeling the Earth's Climate System, Technical Note NCAR/TN-409+PROC, NCAR, Colorado (1994).

13. Beran, J.: Statistics for Long Memory Processes. Chapman and Hall/CRC, Boca Raton (1998).

14. Franzke, C. L. E., Barbosa, S., Blender, R., Fredriksen, H.-B., Laepple, T., Lambert, F., et al.: The structure of climate variability across scales. Reviews of Geophysics. 58, e2019RG000657 (2020). doi:10.1029/2019RG000657 
15. Rypdal, K: Global temperature response to radiative forcing: Solar cycle versus volcanic eruptions. J. Geophys. Res. Atmos. 117, D06115 (2012).

16. Fredriksen, H.-B., Rypdal, M.: Long-range persistence in global surface temperatures explained by linear multibox energy balance models. Journal of Climate. 30(18), 7157-7168 (2017).

17. Rypdal, M., Fredriksen, H.-B., Myrvoll-Nilsen, E., Rypdal, K., Sorbye, S. H.: Emergent scale invariance and climate sensitivity. Climate. 6(4), 93 (2018). doi:10.3390/cli6040093

18. Del Rio Amador, L., Lovejoy, S.: Predicting the global temperature with the Stochastic Seasonal to Interannual Prediction System (StocSIPS). Climate Dynamics (2019). doi:10.1007/s00382-019-04791-4

19. Mori, H.: Transport, collective motion and Brownian motion. Progress of Theoretical Physics. 33(3), 423-455 (1965). doi:10.1143/PTP.33.423.

20. Coffey, W. T., Kalmykov, Y. P.: The Langevin Equation: With Applications to Stochastic Problems in Physics, Chemistry and Electrical Engineering, 4th Edition. World Scientific, Singapore (2017).

21. Chechkin, A. V., Klages, R.: Fluctuation relations for anomalous dynamics. Journal of Statistical Mechanics. L03002/1-11 (2009)

22. Chechkin, A. V., Lenz, F., Klages, R.: Normal and anomalous fluctuation relations for Gaussian stochastic dynamics. Journal of Statistical Mechanics. L11001/1-13 (2012)

23. Lovejoy, S.: Weather, Macroweather, and the Climate: Our Random Yet Predictable Atmosphere. Oxford University Press, Oxford (2019).

24. Lovejoy, S.: Fractional relaxation noises, motions and the fractional energy balance equation. Nonlinear Processes in Geophysics Discussions. 1-52 (2019). doi:10.5194/npg-2019-39

25. Lovejoy, S., Procyk, R., Hébert, R., Del Rio Amador, L.: The Fractional Energy Balance Equation. In review, Quarterly Journal of the Royal Meteorological Society. http://www.physics.mcgill.ca/ gang/eprints/eprintLovejoy/esubmissions/QJRMS.FEBE.revised.3.11.20.pdf

26. Lemons, D. S.: An Introduction to Stochastic Processes in Physics. Johns Hopkins University Press, Baltimore (2002).

27. Cox, P. M., Huntingford, C., Williamson, M. S.: Emergent constraint on equilibrium climate sensitivity from global temperature variability. Nature. 553, 319-322 (2018) doi:10.1038/nature25450

28. Gardiner, C. W.: Handbook of Stochastic Methods: For Physics, Chemistry and the Natural Sciences, 3rd Edition. Springer Verlag, Berlin (2004).

29. Hänggi, P., Jung, P: Colored Noise in Dynamical Systems. Advances in Chemical Physics. Vol LXXXIX. Prigogine, I, Rice, S. A (eds.). Wiley (1995).

30. Lutz, E.: Fractional Langevin equation. Physical Review E, 64, 051106 (2001).

31. Kondrashov, D., Chekroun, M. D., Ghil, M.. Data-driven non-Markovian closure models. Physica D, 297, 33-55 (2015).

32. Darve, E., Solomon, J., Kia, A.: Computing generalized Langevin equations and generalized Fokker-Planck equations. Proceedings of the National Academy of Sciences of the United States of America. 106(27), 10884-10889 (2009).

33. Porrà, J. M., Wang, K. G., Masoliver, J.: Generalized Langevin equations: Anomalous diffusion and probability distributions. Physical Review E. 53(6), 2044-2056 (1996).

34. Mainardi, F., Pironi, P.: The fractional Langevin equation: Brownian motion revisited. Extracta Mathematicae. 11 , 140-154 (1996). 
35. Molina-Garcia, D., Sandev, T., Safdari, H., Pagnini, G., Chechkin, A., Metzler, R.: Crossover from anomalous to normal diffusion: truncated power-law noise correlations and applications to dynamics in lipid bilayers. New Journal of Physics. 20, 103027 (2018). doi:10.1088/1367-2630/aae4b2

36. Kobelev, V., Romanov, E.: Fractional Langevin equation to describe anomalous diffusion. Progress of Theoretical Physics Supplement. 139, 470-476 (2000).

37. Metzler, R., Jeon, J., Cherstvy, A. G., Barkai, E.: Physical Chemistry Chemical Physics. 16, 24128 (2014). doi:10.1039/C4CP03465A

38. Watkins, N. W.: Mandelbrot's stochastic time series models. Earth and Space Science. 6, 2044-2056 (2019). doi:10.1029/2019EA000598

39. https://meetingorganizer.copernicus.org/EGU2019/presentations/watkins

40. https://www.essoar.org/doi/abs/10.1002/essoar.10501367.1 